This is an Original Manuscript of an article published by Taylor \& Francis in Philosophical Magazine on 2017, available at http://www.tandfonline.com/doi/full/10.1080/14786435.2016.1263402.

\title{
Softening and Hardening of Yield Stress by Hydrogen-Solute Interactions
}

\author{
A. Tehranchi, B. Yin, and W.A. Curtin \\ Laboratory for Multiscale Mechanics Modeling, EPFL, CH-1015 Lausanne, Switzerland
}

\begin{abstract}
ARTICLE HISTORY
Compiled August 3, 2016

ABSTRACT

Hydrogen atoms have a wide variety of effects on the mechanical performance of metals, and the underlying mechanisms associated with effects on plastic flow and embrittlement remain to be discovered or validated. Here, the reduction in the plastic flow stress (softening) due to hydrogen atoms in solute-strengthened metals, previously proposed by Sofronis et al., is demonstrated at the atomistic level. Glide of an edge dislocation through a field of solutes in a Nickel matrix, both in the absence of hydrogen and in the presence of $\mathrm{H}$ bound to the solutes, is modeled. The "solutes" here are vacancies, enabling use of available binary $\mathrm{Ni}-\mathrm{H}$ interatomic potentials, but vacancies have a misfit strain tensor in the Ni matrix and bind Hydrogen atoms, and so are excellent surrogates for study of the general phenomenon. The binding of $\mathrm{H}$ to a vacancy reduces the misfit volume to nearly zero but also creates a non-zero tetragonal distortion. Solute strengthening theory is used to establish the connection between strength and solute/hydrogen concentration and misfit strain tensor. Simulations show that when a dislocation moves through a field of random vacancy "solutes", the glide stress is reduced (softening) when H is bound to the solutes. Trends in the simulations are consistent with theory predictions. Trends of softening or hardening by $\mathrm{H}$ in metal alloys can thus be made by computing the misfit strain tensor for a desired solute in the chosen matrix with and without bound Hydrogen atoms. Pursuing this, density functional theory calculations of the interaction of $\mathrm{H}$ with Carbon and Sulfur solutes in a Ni matrix are presented. These solutes/impurities do not bind with $\mathrm{H}$ and the complexes have larger misfit strains, indicative of $\mathrm{H}$-induced strengthening rather than softening for these cases. Nonetheless, $\mathrm{H}$ /solute interactions are the only mechanism that, to date, shows nanoscale evidence of plastic softening due to Hydrogen associated with the Hydrogen-Enhanced Localized Plasticity (HELP) concept.
\end{abstract}

\section{KEYWORDS}

Solute strengthening; Dislocation; Softening; Hydrogen

\section{Introduction}

The detrimental effects of hydrogen atoms on the mechanical properties of metals has been known for over a century [1. One of the most important effects in metal alloys based on Iron and Nickel is embrittlement [2 7], i.e. premature fracture or re5 duced ductility. Although intense scientific effort has been devoted to understanding different aspects of hydrogen embrittlement (HE), there is still no consensus on the mechanisms causing the behavior, nor any general theory that can describe the many 
effects of $\mathrm{H}$ on metal flow and fracture. Nonetheless, there are a few theories that can partially capture some features of HE. Hydrogen enhanced decohesion (HEDE) postulates that $\mathrm{H}$ atoms reduce the surface energy of cleavage surfaces, reducing the applied stress intensity required for cleavage fracture 8-13]. A related mechanism involves the aggregation of $\mathrm{H}$ around the crack tip that prevents emission of dislocations from the crack tip, thus preventing blunting and allowing cleavage fracture to occur. This mechanism depends on the rate of hydrogen diffusion to the crack tip, and a model that incorporates both the crack-tip phenomenon and the $\mathrm{H}$ transport successfully predicts embrittlement in ferritic steels [14, 15]. An entirely different concept is HydrogenEnhanced Localized Plasticity (HELP) [16 18], for which several mechanisms have been postulated. The general idea in HELP is that dislocation motion or nucleation is facilitated by $\mathrm{H}$, leading to either more dislocations, more localized pile-ups, or more 20 extensive plastic straining around a crack tip. The connection to embrittlement is not direct, but macroscopic experiments show that $\mathrm{H}$ can lead to plastic softening and to higher dislocation activity near crack tips or fracture surfaces. In-situ TEM experiments also show that $\mathrm{H}$ affects dislocation motion, although the mechanisms are not clearly identified. Sofronis and coworkers examined possible mechanisms using various 25 continuum models. Both experiments and theory concluded that $\mathrm{H}$ does not influence long-range dislocation-dislocation interactions, eliminating one mechanism; this was recently supported by direct atomistic simulations [19, 20]. However, there is some evidence that the presence of $\mathrm{H}$ atoms can have significant influences on the interaction between pre-existing solutes and dislocations, thus modifying the plastic flow stress.

30 Birnbuam and Sofronis [17] performed a finite element analysis to estimate the effect of the $\mathrm{H}$ atoms on the interaction energy between interstitial carbon atoms and an edge dislocations in $\mathrm{Nb}$ and found a decrease of $0.5 \mathrm{eV}$ in the maximum interaction. While no direct connection to lower flow stresses was made, a reduction would be expected.

35 Many advanced engineering metals contain solutes, either for direct solute strengthening or as a residual of precipitate strengthening. In both cases, there is a contribution to the flow stress from the solutes in the matrix due to dislocation interactions with the field of solute atoms. A recent predictive model for the finite-temperature, finitestrain rate, solute strengthening by Leyson et al. [21 23] is a parameter-free theory whose only inputs are the specific dislocation/solute interaction energies and assumed random fluctuations of the solute distribution. This theory thus provides the specific guidance needed to connect Hydrogen-Solute interactions to solute strengthening of the host metal. Specifically, if pre-existing solutes can bind hydrogen atoms to form Solute-H complexes, the difference in misfit distortion between the Solute and the Solute-H complex will change the interactions with the dislocations, and raise or lower the flow stress. The purpose of the present work is to clearly demonstrate this mechanism, in the case of softening, using direct atomistic simulations as guided by solute strengthening theory. For simplicity in dealing with "chemical" interactions among multiple atom types, we use vacancies (V) as the "solutes" in $\mathrm{Ni}$, which enables the 50 use of a well-established $\mathrm{Ni}-\mathrm{H}$ binary potential for the study of the $\mathrm{Ni}-\mathrm{H}-\mathrm{V}$ system. We show that $\mathrm{H}$ modifies the misfit strain tensor, and that an individual dislocation moves through a field of $\mathrm{V}-\mathrm{H}$ complexes at lower stresses than through a field of $\mathrm{V}$ in exactly the same random configuration. The scaling of the effects with $\mathrm{V}$ concentration follow the predictions of the theory, verifying the origins of the strengthening 55 of the vacancy solutes and the softening due to the introduction of $\mathrm{H}$ bound to the vacancies. As a preliminary step toward realistic situations, we then use first-principles calculations based on density functional theory to study the interaction of $\mathrm{H}$ atoms 
with the pre-existing Sulfur and Carbon solutes in Ni. These cases show H-Solute repulsion and increases in misfit strain tensor, thus indicating no softening, but serve to demonstrate the approach for general H-Solute-Matrix studies. The overall results and analysis here provide a path for understanding softening and hardening due to $\mathrm{H}$ in metal alloys.

The remainder of this paper is organized as follows. In Section 2, the theory of solute strengthening for straight dislocations is adapted to the problem of interstitial solutes with tetragonal misfit distortions. In Section 3 , the details of atomistic simulations of dislocation motion through a field of vacancies and vacancies with bound $\mathrm{H}$, with the same spatial distribution of vacancies, are presented. Section 4 presents the results of the simulations and discussion of the softening effect of $\mathrm{H}$. Section 5 presents a density functional theory (DFT) study of the interaction Carbon and Sulfur solute atoms with

$70 \mathrm{H}$, including computation of the misfit strain tensors. A summary of our findings is given in Section 6 .

\section{Theoretical framework}

The theory for solute strengthening is well-established in a sequence of recent publications $21-23$. Here, we introduce the key features and results of the theory, without a complete derivation and generalize the theory to include tetragonal misfit distortions.

We consider a straight edge dislocation with Burgers vector $\mathbf{b}=b \mathbf{e}_{1}$ lying along the $x_{3}$-axis in an isotropic medium with shear modulus $\mu$ and Poisson's ratio $\nu$ containing $M$ different types of solutes with concentrations $c_{m}(m=1,2, \ldots, M)$ distributed randomly throughout the matrix, as shown in Fig. 1. To take advantage of local fluctuations in the solute concentration that arise naturally in the random solution, the straight dislocation will roughen so as to move segments into local fluctuations where the particular spatial distribution of solutes leads to a net binding, i.e. lowering of the energy, of the solute/dislocation system (see Fig. 2). There is an energy cost to roughening due to the elastic interactions of the non-straight dislocation and its increase in length, which is captured through the dislocation line tension. The dislocation will thus adopt a particular configuration corresponding to a characteristic amplitude $w_{c}$ and characteristic wavelength $\zeta_{c}$ that minimize the total energy of the long dislocation $\left(L>>\zeta_{c}\right)$ [21 23]. The local bound dislocation segments of length $\zeta_{c}$ reside in local energy minima of width $w_{c}$, and a combination of applied stress and temperature is necessary for the segments to escape these local minima by thermal activation. Given the solute/dislocation interaction energy as a function of solute position relative to the dislocation, the theory predicts $w_{c}, \zeta_{c}$, the energy barrier $\Delta E_{b}$, and the zero-temperature flow stress $\tau_{y 0}$, from which thermal activation theory leads to the finite- $\mathrm{T}$, finite strain rate flow stress $\tau_{y}$.

The most important feature in solute strengthening theory is the interaction energy of a dislocation with the solute atoms. Here, we confine the analysis to elastic interactions only. In the elastic case, the interaction energy is equal to the work done by the dislocation stress field $\boldsymbol{\sigma}$ over a the deformation corresponding to the misfit strain tensor $\varepsilon$ for the solute. For a dislocation segment of length $\zeta$ located at the origin lying in a specified random configuration of solutes, the total interaction energy is

$$
E_{\mathrm{int}}(\zeta)=\sum_{i j k} \sum_{m} s_{i j k}^{(m)} \boldsymbol{\sigma}\left(x_{1}^{(i)}, x_{2}^{(j)}\right): \boldsymbol{\varepsilon}^{(m)}
$$


with $s_{i j k}^{(m)}=1$ if a type-m solute exists at position $\left(x_{1}^{(i)}, x_{2}^{(j)}, x_{3}^{(k)}\right)$ and 0 otherwise. Here, $\boldsymbol{\sigma}$ is the tensorial stress field induced by the dislocation at the location of the solute and $\boldsymbol{\varepsilon}^{(m)}$ is the misfit strain tensor of the solute of type $m$, and $\boldsymbol{\sigma}: \boldsymbol{\varepsilon}=\sigma_{i j} \varepsilon_{i j}$ indicates the contraction of the two tensors. If the dislocation segment glides a distance $w$ along the glide direction $\mathbf{e}_{1}$, the change in the interaction energy is

$$
\begin{aligned}
\Delta E_{\mathrm{int}}(\zeta, w) & =\sum_{i j k} \sum_{m} s_{i j k}^{(m)}\left(\boldsymbol{\sigma}\left(x_{1}^{(i)}-w, x_{2}^{(j)}\right)-\boldsymbol{\sigma}\left(x_{1}^{(i)}, x_{2}^{(j)}\right)\right): \boldsymbol{\varepsilon}^{(m)} \\
& =\sum_{i j k} \sum_{m} s_{i j k}^{(m)} \Delta \boldsymbol{\sigma}\left(x_{1}^{(i)}, x_{2}^{(j)} ; w\right): \boldsymbol{\varepsilon}^{(m)} \\
& =\sum_{i j} \sum_{m} \mathbf{n}_{i j}^{(m)} \Delta \boldsymbol{\sigma}\left(x_{1}^{(i)}, x_{2}^{(j)} ; w\right): \boldsymbol{\varepsilon}^{(m)}
\end{aligned}
$$

where $\mathbf{n}_{i j}^{(m)}=\sum_{k} s_{i j k}^{(m)}$ is the number of solutes type-m at an atomic row lies on a line segment of length $\zeta$ parallel to the dislocation line passing through $\left(x_{1}^{(i)}, x_{2}^{(j)}\right)$. The key quantity in the solute strengthening model is the standard deviation of the total interaction energy change

$$
\sigma_{\Delta E_{\text {int }}}^{2}(\zeta, w)=\left\langle\Delta E_{\text {int }}(\zeta, w)^{2}\right\rangle-\left\langle\Delta E_{\text {int }}(\zeta, w)\right\rangle^{2} .
$$

95 After extensive manipulations and in the dilute concentration limit for all solute types (i.e. $\left.c_{m} \ll 1, \quad m=1,2, \ldots, M\right)$, the standard deviation of the energy change is

$$
\sigma_{\Delta E_{\mathrm{int}}}^{2}(\zeta, w)=\frac{\zeta}{\sqrt{3} b} \Delta \tilde{E}_{p}=\frac{\zeta}{\sqrt{3} b} \sum_{i j} \sum_{m}\left(\Delta \boldsymbol{\sigma}\left(x_{1}^{(i)}, x_{2}^{(j)} ; w\right): \varepsilon^{(m)}\right)^{2} c_{m}
$$

The standard deviation above is the typical energy scale for binding of the dislocation to the random solutes. From this quantity, the solute strengthening theory derives all other quantities of interest, culminating in the finite- $T$, finite strain rate flow stress.

For the present atomistic study, we are interested only in the motion of a single straight dislocation of some length $\zeta<\zeta_{c}$, which should remain straight since the energy cost to bow out is too high. The straight dislocation is pinned in regions where the energy is $-\sigma_{\Delta E_{\text {int }}}$ and must be thermally activated over regions where the energy is $+\sigma_{\Delta E_{\text {int }}}$, with these maxima and minimum being separated by $w_{c}$. That is, the single segment is envisioned as moving through an energy landscape that is locally sinusoidal.

$$
E\left(\zeta, x_{1}\right)=\frac{\Delta E_{b}^{\prime}}{2}\left[1-\cos \left(\frac{\pi x}{w_{c}}\right)\right]-\tau \zeta b x_{1}
$$

where $\Delta E_{b}^{\prime}=\sqrt{2} \sigma_{\Delta E_{\text {int }}}$ is the energy barrier. Under an applied resolved shear stress $\tau$ that drives the dislocation and further lowers the energy as a function of glide distance $x_{1}$, the energy landscape becomes

$$
E\left(\zeta, x_{1}\right)=\frac{\Delta E_{b}^{\prime}\left(\zeta, w_{c}\right)}{2}\left[1-\cos \left(\frac{\pi x}{w_{c}}\right)\right]-\tau \zeta b x_{1}
$$




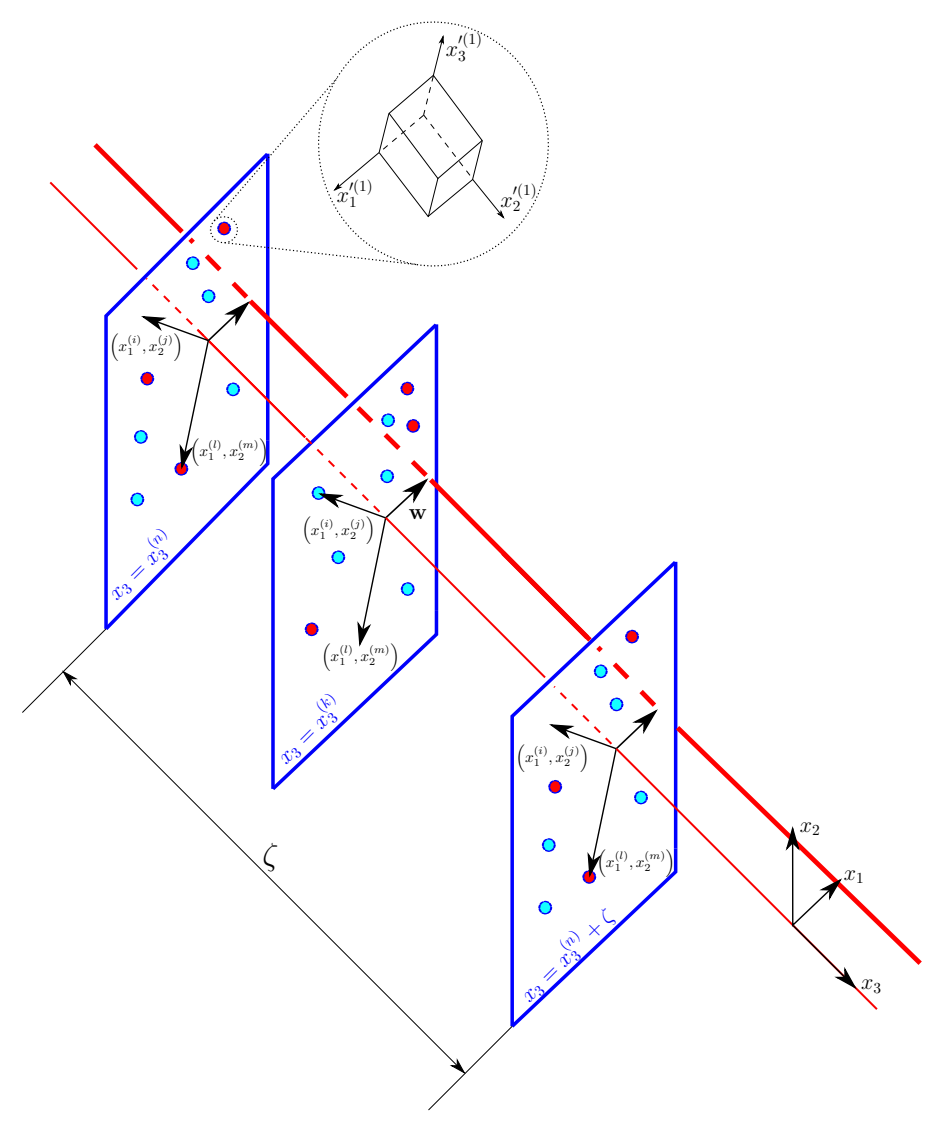

Figure 1.: An edge dislocation originally lying on the $x_{3}$ axis surrounded by the multiple solutes of different types. The red and blue circles refer to two types of solute atoms. The principal misfit strain coordinate system of the solutes are $\left\{x_{i}^{\prime(m)}\right\}$. The dislocation glides a distance $w$ along the $x_{1}$ axis.

The zero-temperature flow stress $\tau_{y 0}$ is the stress at which the barrier in the total energy landscape becomes zero. This is obtained analytically from Eq. (6) and is given by

$$
\tau_{y 0}=\frac{\pi}{2} \frac{\Delta E_{b}^{\prime}}{b w_{c} \zeta}=-\frac{\pi}{w_{c} \sqrt{2}}\left(\frac{1}{\zeta \sqrt{3} b^{3}}\right)^{\frac{1}{2}} \sqrt{\sum_{i j} \sum_{m}\left(\Delta \boldsymbol{\sigma}\left(x_{1}^{(i)}, x_{2}^{(j)} ; w_{c}\right): \boldsymbol{\varepsilon}^{(m)}\right)^{2} c_{m}}
$$

where we have substituted the expression for $\sigma_{\Delta E_{\text {int }}}$. Eq. (7) is the theoretical result relevant to the simulations discussed below, providing the relationship between the underlying solute/dislocation interaction energies, here assumed to be only elastic, and the typical stress required to move a dislocation through a field of such solutes. The original theory does not rely on the elasticity assumption and uses full DFTcomputed solute/dislocation interaction energies, but that is beyond the scope of the present work.

The misfit strain tensor of a solute or solute complex can, in general, be separated into volumetric and deviatoric components. Written in its principal coordinate 


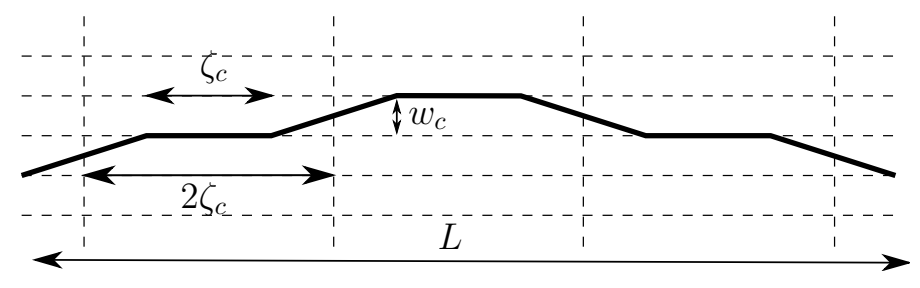

Figure 2.: Schematic diagram of a dislocation segment of length $L$ roughened into segments of length $2 \zeta_{c}$ with amplitude $w_{c}$, due to the spatial fluctuations of the solutes within the surrounding medium.

$\left\{x_{i}^{\prime(m)}\right\} \quad(i=1,2,3)$, the misfit strain tensor is

$$
\varepsilon^{\prime(m)}=\varepsilon_{d}^{\prime(m)}+\varepsilon_{v}^{(m)}
$$

where

$$
\varepsilon_{d}^{\prime(m)}=\frac{\delta V^{(m)}}{3 V}\left(\begin{array}{ccc}
\frac{2-\xi-\eta}{1+\xi+\eta} & 0 & 0 \\
0 & \frac{2 \xi-1-\eta}{1+\xi+\eta} & 0 \\
0 & 0 & \frac{2 \eta-\xi-1}{1+\xi+\eta}
\end{array}\right)
$$

and

$$
\boldsymbol{\varepsilon}_{v}^{(m)}=\frac{\delta V^{(m)}}{3 V}\left(\begin{array}{lll}
1 & 0 & 0 \\
0 & 1 & 0 \\
0 & 0 & 1
\end{array}\right)
$$

with $\delta V^{(m)} / V$, the misfit volumetric strain, and $\xi$ and $\eta$ the ratios of the second and third principal strains to the first principal strain, respectively. The stress field induced by the dislocations can also be expressed in terms of volumetric and deviatoric contributions as

$$
\boldsymbol{\sigma}\left(x_{1}, x_{2}\right)=\boldsymbol{\sigma}^{\prime}\left(x_{1}, x_{2}\right)+\boldsymbol{p}\left(x_{1}, x_{2}\right)
$$

Inserting these decompositions into Eq. (7), we obtain

$$
\tau_{0}=-\frac{\pi}{w_{c} \sqrt{2}}\left(\frac{1}{\zeta \sqrt{3} b^{3}}\right)^{\frac{1}{2}} \sqrt{\sum_{i j} \sum_{m}\left(\left(\Delta \boldsymbol{\sigma}^{\prime}\left(x_{1}^{(i)}, x_{2}^{(j)} ; w_{c}\right): \varepsilon_{d}^{(m)}\right)^{2}+\Delta p^{2}\left(x_{1}^{(i)}, x_{2}^{(j)} ; w_{c}\right)\left(\frac{\delta V^{(m)}}{V}\right)^{2}\right) c_{m}}
$$

There are thus two contributions to the strengthening, a volumetric piece due to the misfit volumes of the solutes and a deviatoric piece due to tetragonal strains. For substitutional solutes, the deviatoric part is zero by symmetry, and was not previously considered. We note 110 also that the stress field entering the strengthening is the difference in stress field between the dislocation at $w_{c}$ and at the origin.

Specific results emerge by using the true stress field of the dislocation. In fcc matrices, the edge dislocation dissociates into two partials separated by a stacking fault, and so the 
classical Volterra solution for singular dislocation is inappropriate. Furthermore, the value for

$115 w_{c}$ is not specified here, and emerges from detailed computations. Here, we focus not on specific predictions but on the major trends predicted by the model. Specifically, the model shows that the strengthening is controlled by contributions from both volumetric and deviatoric strains, and that the solute concentrations enter within a sum that is within an overall square-root. If all solutes have the same concentration $c$, the strengthening of a straight dislocation moving

120 through the random field of solutes scales as $c^{1 / 2}$; this scaling is different from that for the long roughened dislocation where the strength scales as $c^{2 / 3}$ due to the difference in geometry. The present model, with $c^{1 / 2}$ scaling, is only appropriate for the case of short segments $\zeta<\zeta_{c}$ considered in the simulations here.

\section{Atomistic Simulation Methodology}

125 We use a simulation cell comprised of a rectangular fcc Ni lattice oriented as $\hat{\mathbf{e}}_{1}=\frac{1}{\sqrt{2}}[10 \overline{1}]$, $\hat{\mathbf{e}}_{2}=\frac{1}{\sqrt{3}}[111], \hat{\mathbf{e}}_{3}=\frac{6}{\sqrt{3}}[1 \overline{2} 1]$. The dimensions of the simulation cell are $L_{1}=503 \AA, L_{2}=$ $306 \AA$ and $L_{3}=155 \AA$. The simulation cell is periodic in the $x_{1}$ and $x_{2}$ directions and has free boundaries in the $x_{3}$ direction. Loading is then accomplished by apply a shear traction $\tau_{12}^{\text {app }}=\tau^{\text {app }}$ to top and bottom (normal vectors $+/-x_{3}$ ) of the specimen.

130 As noted earlier, we use vacancies as the "solutes" in this problem. To insert vacancies with desired concentrations, we randomly delete $N_{\mathrm{v}}=c N$ of the $N$ Ni atoms in the simulation cell. However, we do not insert any vacancies along the two atomic planes above and two atomic planes below the dislocation (111) glide plane. This is a special restriction for the vacancies because, unlike solutes, vacancies can have unusual interactions with the dislocation core. In

135 particular, randomly occurring vacancy clusters can lead to the formation of dislocation jogs, which are strengthening obstacles. The special exclusion of vacancies from several atomic rows has no consequences for the form of the theory in Eq. (9) since the summation is restricted to sites where the vacancies exist. In fact, eliminating solute/core and solute/stacking fault interactions also makes the elastic interactions dominant. After deleting the Ni atoms, we relax

140 the specimen with standard conjugate gradient method [24]. For every specimen containing a particular spatial distribution of vacancies, we create a second specimen in which we insert one hydrogen atom at each vacancy site and then let relax the system again. During relaxation, the hydrogen moves to one of the symmetry-related low-energy positions associated with the vacancy. Thus, we have two samples with identical spatial distributions of either solutes (V) 45 or solute-hydrogen (V-H) complexes.

After initial relaxation, we insert an edge dislocation of Burgers vector $\frac{a}{2}[10 \overline{1}]$, where $a=$ $3.52 \AA$ is the lattice constant, and line direction [12 1$]$ along the $x_{3}$-axis at the center of the specimen $x_{1}=X_{0}$. We use the standard procedure [25] of removing the atoms with coordinates $-\frac{b}{2}<x_{1}-X_{0}<b / 2, x_{3}<L_{2} / 2$ from the perfect crystal followed by relaxation of the 150 system with the conjugate gradient method. During relaxation, a dislocation is formed and it dissociates into two partial dislocations with Burgers vectors $\frac{a}{6}[\overline{1} \overline{1} 2]$ and $\frac{a}{6}[2 \overline{1} \overline{1}]$. The geometric details of the simulation cell are shown in Fig. 3 .

The MD simulations are performed in the NVT ensemble using a Langevin thermostat 26 and the velocity-Verlet algorithm [27] with integration time step of 1 fs. The temperature of

155 the system is fixed to be $10^{-3} K$ since we are not probing thermally-activated flow here. The interatomic interactions among atoms are described using the $\mathrm{Ni}-\mathrm{H}$ embedded atom method (EAM) potential introduced by Angelo et al. [28] and modified by Song and Curtin [14. The shear stress is increased from $\tau_{12}^{\text {app }}=0$ in increments of $\Delta \tau=1 \mathrm{MPa}$. After each loading step, $\mathrm{MD}$ is executed for $\Delta t=30 \mathrm{ps}$ and then the $x_{1}$-coordinate of the dislocation core, defined as

160 the centerline of the stacking fault between two partials, is compared to that of the previous step. If the incremental displacement of the dislocation core is larger than $\Delta d=1.1 \AA$, then the MD simulation is executed for another period of $\Delta t$. Otherwise, the load is increased by $\Delta \tau$. During the simulation, we determine the dislocation position along the glide plane versus the applied stress $\tau^{\text {app }}$. We perform loading steps until the dislocation has passed through the 65 entire periodic length $L_{x}$ of the glide plane in the simulation cell. The applied stress $\tau^{\text {app }}$ at this point of the simulation is taken as a measure of the flow stress.

The molecular dynamics simulations are performed using the Large-scale Atomic/Molecular 


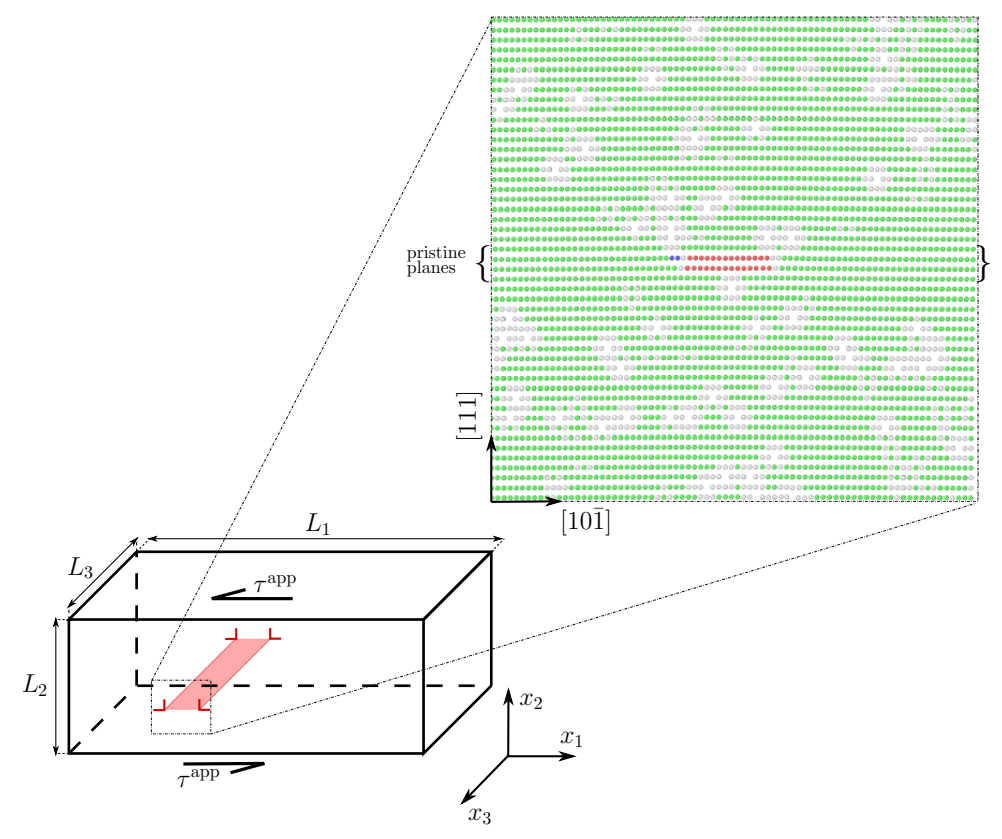

Figure 3.: Schematic diagram of the simulation cell containing the straight dislocation and vacancies subjected to the applied shear traction. The color coding for the atomistic picture uses common neighbor analysis (green: fcc; red: hcp indicating the stacking fault; white: atoms having fewer than 12 nieghbors and thus surrounding a vacancy.

Massively Parallel Simulator (LAMMPS) 29] and atomic configurations are visualized using the Open Visualization Tool (OVITO) 30].

\section{Results and discussion}

We have measured the values for the misfit strain tensor quantities (misfit volume, $\xi, \eta$ ) and their principal directions for an isolated vacancy $\mathrm{V}$ and for an isolated $\mathrm{VH}$ complex for the $\mathrm{Ni}-\mathrm{H}$ EAM potential used here, as shown in Table 1 . Note that the maximum principal strain associated with $\mathrm{VH}$ can be oriented along one of three distinct crystallographic directions.

175 We consider these differently-oriented VHs as different types of solutes, since they interact differently with the dislocation; we denote the three types of complexes as $\mathrm{VH}^{(m)},(m=1,2,3)$ with equal concentrations $c_{m}=c / 3 \quad(m=1,2,3)$ where $c$ is the $\mathrm{V}$ concentration. For the $\mathrm{V}$, the misfit strain is purely volumetric and so the interaction energy only involves the dislocation pressure field. For the VH complexes, the volumetric term is nearly zero but the deviatoric

180 strains are not negligible, so that the interaction energy is dominated by the deviatoric field of the dislocation. Thus, there is a fundamental change in the nature of the dislocation/vacancy interactions upon the introduction of $\mathrm{H}$ to form VH. Fig. 4 illustrates the angular variation of the normalized deviatoric and volumetric components of the interaction energy density for a $\mathrm{V}$ and each $\mathrm{VH}^{(m)}$ complex as computed by Eqs. (10) for a singular Volterra dislocation.

185 Clearly, the angular dependency of the interaction energy of VH is different from that of V. Most importantly, the maximum interaction energy is reduced by $44 \%$ by the addition of $\mathrm{H}$. Thus, the theory predicts that Hydrogen should have a significant softening effect (lower stress to move a dislocation) when interacting with suitable solutes. Considering that (i) a realistic dislocation is dissociated into two partials, (ii) the largest interaction energies occur close to

190 the core, and (iii) the theory depends on the difference in dislocation stress field at $w_{c}$ and at the origin (with $w_{c}$ typically emerging as comparable to the dissociation distance), the angular 
This is an Original Manuscript of an article published by Taylor \& Francis in Philosophical Magazine on 2017, available at http://www.tandfonline.com/doi/full/10.1080/14786435.2016.1263402.

dependence shown in Fig. 4 does not translate directly into a precise prediction for the change in flow stress upon addition of $\mathrm{H}$. But a softening effect is expected due to the overall reduction in interaction energies. We next show this explicitly in the atomistic simulations.

Table 1.: Parameters describing the misfit strain tensor of each type of solutes.

\begin{tabular}{lcccccc}
\hline solute & $\xi$ & $\eta$ & $\frac{\delta V}{V}$ & $\mathbf{e}_{1}^{\prime}$ & $\mathbf{e}_{2}^{\prime}$ & $\mathbf{e}_{3}^{\prime}$ \\
\hline \hline $\mathrm{VH}^{(1)}$ & 1.0 & -1.7857 & 0.00836 & {$[100]$} & {$[010]$} & {$[001]$} \\
$\mathrm{VH}^{(2)}$ & 1.0 & -1.7857 & 0.00836 & {$[010]$} & {$[001]$} & {$[100]$} \\
$\mathrm{VH}^{(3)}$ & 1.0 & -1.7857 & 0.00836 & {$[001]$} & {$[100]$} & {$[010]$} \\
$\mathrm{V}$ & 1.0 & 1.0 & -0.16168 & - & - & - \\
\hline
\end{tabular}

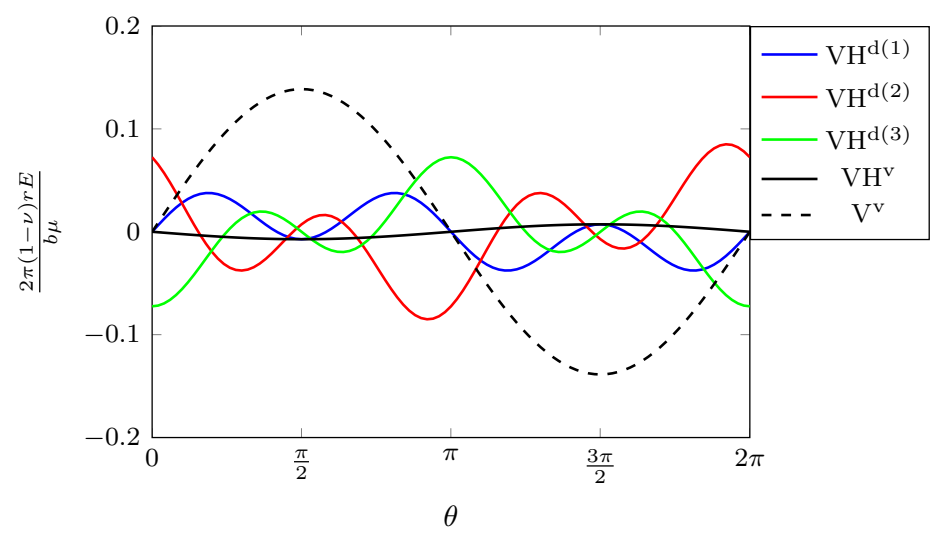

Figure 4.: Normalized volumetric and deviatoric parts of the interaction energy as a function of polar angle around a Volterra edge dislocation, for different orientations of the VH solute complexes studied here.

To demonstrate the expected softening effect of hydrogen, we study specimens with a range of $\mathrm{V}$ or $\mathrm{VH}$ concentrations from $0.5 \%$ to $3.5 \%$. These are realistic concentrations for real solutes in metal alloys, recalling that the vacancies are surrogates for solutes. The corresponding concentration of $\mathrm{VH}$ complexes implies that the $\mathrm{H}$ concentration is $0.5 \%$ to $3.5 \%$, which is much higher than nominal concentrations at which $\mathrm{H}$ embrittlement is observed. However, elevated $\mathrm{H}$ concentrations could be anticipated around crack tips. Dislocations themselves can transport H via "solute drag", and so the dislocation plasticity around a crack may itself serve as a means to provide $\mathrm{H}$ that can bind to solutes. Thus, our studies here, while meant to be illustrative of the phenomenon, are within the scope of realistic material systems given mechanisms of local enhancement of the $\mathrm{H}$ concentration.

205 Fig. 5 5hows the dislocation position versus applied shear stress shear as obtained from the MD simulations for specimens with different concentrations of V and VH complexes. Multiple random solute arrangements are shown for each average concentration. For each random solute arrangement, two curves are shown corresponding to the cases without (solid lines) and with (dashed lines) H. In every specimen, the stress at any given dislocation position is always 210 lower in the presence of $\mathrm{H}$. That is, in every specimen, $\mathrm{H}$ softens/reduces the flow stress of that specific specimen. This is the main result of this paper. 
This is an Original Manuscript of an article published by Taylor \& Francis in Philosophical Magazine on 2017, available at http://www.tandfonline.com/doi/full/10.1080/14786435.2016.1263402.

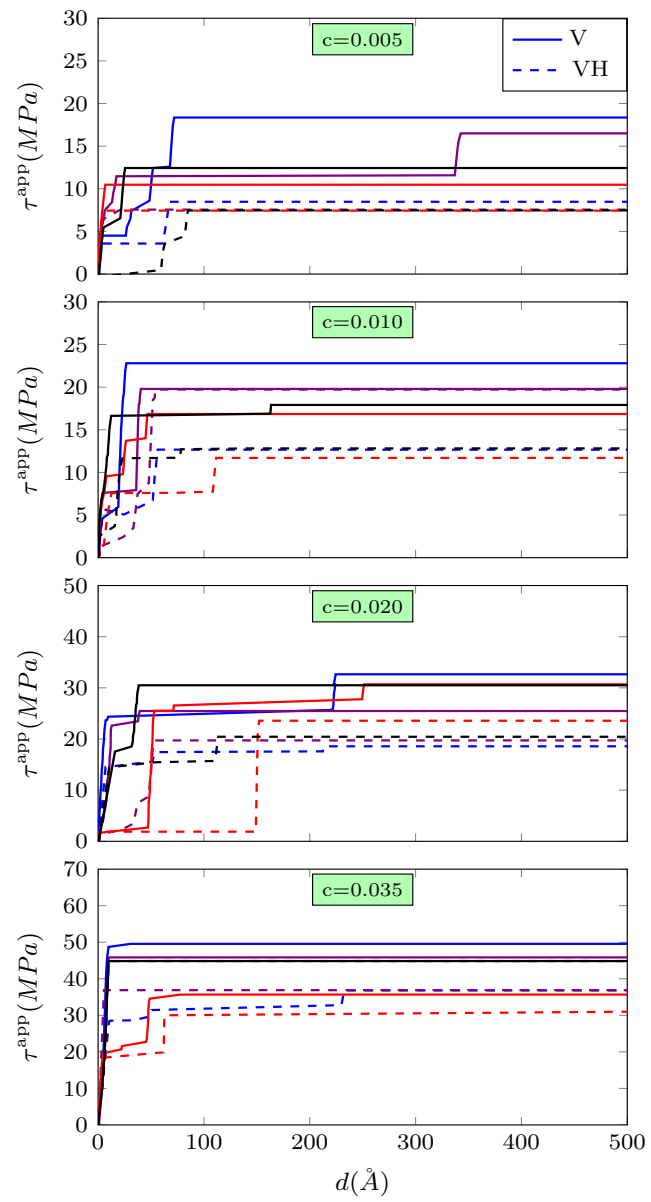

Figure 5.: Applied shear stress $\tau^{\text {app }}$ versus dislocation glide distance $d$, for a several concentrations of V (solid lines) and VH (dashed lines) solutes in fcc Nickel. Same-color solid and dashed lines show results for the same spatial distribution of solutes.

The general occurrence of softening is independent of the glide distance. However, the stepwise nature of the stress vs. glide distance found in all specimens requires some remarks. The nominally straight dislocation segment encounters different random solute environments as it glides. It will therefore get pinned at statistically stronger binding regions as it glides. An increasing stress is thus required to overcome each successive barrier. However, since the distribution of barriers is expected to be Gaussian with standard deviation $\sigma_{\Delta E_{\text {int }}}$, stronger binding regions become increasingly unlikely. Thus, at a certain stress level, the dislocation becomes unpinned and does not encounter any stronger-binding region in the remainder of

220 the sample. The statistical distribution of binding regions thus gives rise to the stochastic pinning and depinning, culminating in a final stage of unabated glide. In addition, variations in the location of the pinning points between the otherwise identical $\mathrm{V}$ and $\mathrm{VH}$ specimens arises because of the differences in spatial variations of the interaction energy, as shown for the Volterra dislocation in Fig. (4). Specifically, the specific fluctuations in spatial V distribution

225 that give rise to the pinning in the presence of vacancies only are not necessarily the same as the fluctuations in spatial VH distribution that give rise to pinning in the presence of $\mathrm{H}$.

Turning to a more quantitative assessment of the results, for comparison purposes, we consider the maximum shear stress (the stress at which the dislocation undergoes glide through the remainder of the specimen) as a nominal "strength" that should scale with the predicted

$230 \tau_{y 0}$. Based on arguments above, we expect this strength to be larger than the true strength of a long dislocation undergoing roughening because it corresponds to a pinning strength that 
scales with, but is larger than, $\sigma_{\Delta E_{\text {int }}}$. As guided by the theory, Figure 6 shows the strength as a function of the square root of $\mathrm{V}$ or $\mathrm{VH}$ concentration, with a linear fit at small concentrations as a guide to the eye. At low concentrations, the measured strength scales directly with $c^{1 / 2}$

235 and the samples with $\mathrm{H}$ are clearly statistically weaker than those without $\mathrm{H}$. Thus, there is a distinct softening effect that scales according to the general solute strengthening theory. The deviation from $c^{1 / 2}$ at the highest concentration could be due to the increasing occurrence of divacancies or other $\mathrm{V}$ clusters that modify the effective misfit volume of each solute, but even here the samples with $\mathrm{H}$ are consistently weaker than those without $\mathrm{H}$.

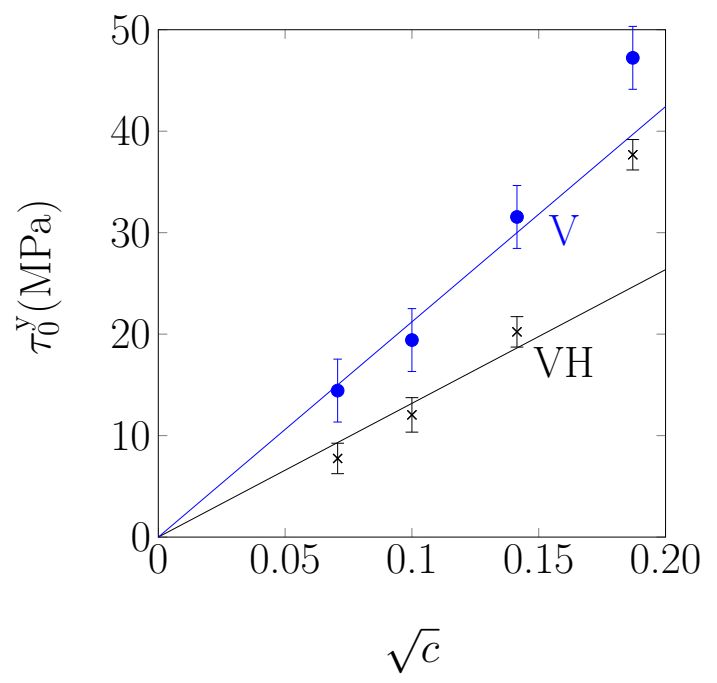

Figure 6.: Shear yield stress $\tau_{y 0}$ as a function of the solute concentration.

The samples with $\mathrm{H}$ are approximately $40 \%$ weaker than the samples without $\mathrm{H}$. This is comparable to the ratio of the root mean square interaction energies for the three VH types of $37 \%$, indicating that the interaction energy change is the dominant factor in the softening. The theory parameter $w_{c}$ is, however, a function of the interaction energies as well and so, in principle, can vary between the $\mathrm{V}$ and $\mathrm{VH}$ systems, leading to some differences in strengthening.

245 Prior results for solute strengthening suggest, however, that variations in $w_{c}$ among different solutes in the same matrix is modest. For instance, in an $\mathrm{Al}$ matrix, $w_{c}=15.7 \AA$ for $\mathrm{Mg}$ (misfit volume $+5.7 \AA^{3}$ ) while $w_{c}=18.6 \AA$ for Mn (misfit volume $-13.3 \AA^{3}$ ) 31 .

Our results here demonstrate, perhaps for the first time, a clear mechanism for the reduction of plastic flow stress due to the presence of hydrogen in a metal. This softening is not necessarily unexpected, from the perspective of solute strengthening theory, but there has been no prior detailed analysis nor simulation study to firmly establish the phenomenon. The analysis here shows, however, that softening is expected only if the overall misfit strain tensor of the Solute-H complex is smaller than the Solute alone. $\mathrm{H}$ has a positive misfit volume, since it is interstitial, and hence solutes that also have positive misfit volumes are unlikely to be

255 "relaxed" by the introduction of $\mathrm{H}$. In general, the presence of $\mathrm{H}$ will likely introduce additional tetragonal distortions, which should also generally contribute to strengthening, since the theory shows that volumetric and deviatoric contributions are generally additive according to a geometric rule. Since, however, the full theory requires computation of the detailed interactions of the realistic dislocation structure with the Solute or Solute-H entities, out of which

260 also emerges characteristic length scales $\zeta_{c}$ and $w_{c}$, it remains possible that softening may not occur even when the changes in misfit strain tensor suggest it. Nonetheless, a combination of solute strengthening theory [21-23] and first-principles calculations of Solute and Solute-H misfit strains can provide solid guidelines for anticipating $\mathrm{H}$-induced softening in general, and estimating the magnitude of the softening. Computation of Solute and Solute- $\mathrm{H}$ misfit strains

265 can be accomplished using moderate-sized unit cells, and so is quite feasible with modern computational power; we discuss this in detail in the next Section. 
This is an Original Manuscript of an article published by Taylor \& Francis in Philosophical Magazine on 2017, available at http://www.tandfonline.com/doi/full/10.1080/14786435.2016.1263402.

\section{Interaction of Carbon and Sulfur solutes with Hydrogen in Nickel}

To demonstrate the general procedure for studying the energetic and mechanical interaction of hydrogen and solute atoms, we analyze the interaction of hydrogen atoms with the pre-existing out using density functional theory (DFT) as implemented in the Vienna $A b$ initio Simulation Package (VASP) 32, 33. Exchange and correlation are treated within the generalized gradient approximation (GGA) with the Perdew-Burke-Ernzerhof (PBE) parametrization 34. Core electrons are replaced by the projector augmented wave (PAW) pseudopotentials [35. The (particle electronic eigenstates are calculated based on a spin-polarized plane-wave set with the cutoff energy of $500 \mathrm{eV}$ and a second-order Methfessel-Paxton method [36] with 0.2 $\mathrm{eV}$ is used to smear the partial occupancies. For the reciprocal space integrations, a $\Gamma$-centered Monkhorst-Pack 37 $k$-point sampling of $28 \times 28 \times 28$ is used for the 4 -atom cubic unit cell and the density of this $k$-point mesh is kept consistent for supercells with larger dimensions. are shown in Fig 7. Based on the parameters mentioned above, $\mathrm{Ni}$ in stress-free bulk state is predicted to have lattice constant and elastic constants of $a=3.524 \AA, C_{11}=273 \mathrm{GPa}$, $C_{12}=157 \mathrm{GPa}$, and $C_{44}=129 \mathrm{GPa}$, all close to the experimental values at $4 \mathrm{~K}\left(C_{11}=261.2\right.$ GPa, $C_{12}=150.8 \mathrm{GPa}$, and $C_{44}=131.7 \mathrm{GPa}$ ) 38, 39. We also obtain a magnetic moment of

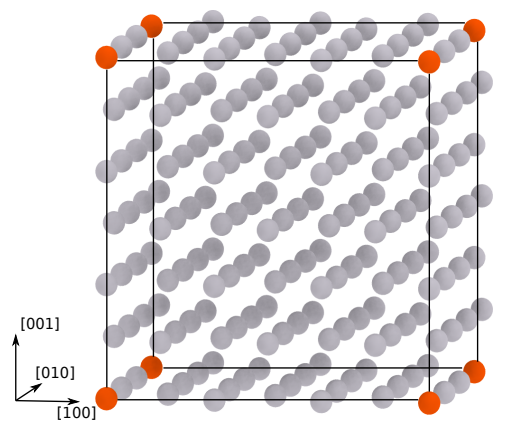

(a) $N i_{107} S$

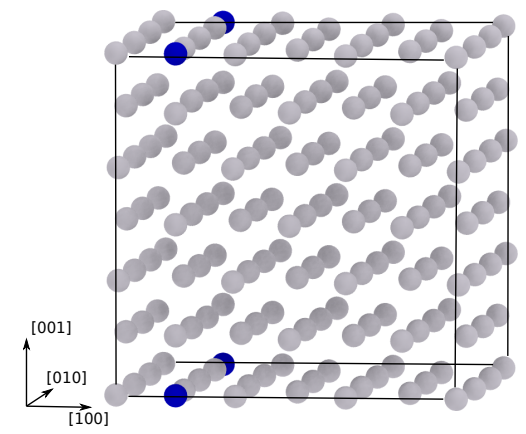

(b) $N i_{108} C$

Figure 7.: Supercells containing (a) a substitutional S solute and (b) an interstitial C solute at the octahedral site. Gray, dark blue, and orange spheres denote the $\mathrm{Ni}, \mathrm{C}$, and $\mathrm{S}$ atoms, respectively.

The interaction energy of $\mathrm{H}$ with a solute $X(\mathrm{X}=\mathrm{C}$ or $\mathrm{S})$ is calculated as

$$
E_{X-H}^{\mathrm{int}}=E_{X-H}-\left(E_{H}+E_{X}-E_{N i}\right),
$$

where $E_{X-H}, E_{X}$ are $E_{H}$ are the energies of supercells containing bulk Ni atoms with a $X-H$, $X$, and $H$ solutes, respectively. $E_{N i}$ denotes the energy of the pure Ni supercell. The interaction energy $E_{X-H}^{\mathrm{int}}$ can be alternatively defined as $E_{X-H^{-}} E^{\text {large }}$, where $E^{\text {large }}$ is the energy of the system with isolated (non-interacting) solutes $\mathrm{X}$ and $\mathrm{H}$ located far from each other. We tested the values of $E_{X-H}^{\mathrm{int}}$ obtained from Eq. (10) against the results of the alternative formulation obtained from supercells of $3 \times 3 \times 3$ and $5 \times 3 \times 3$, in which the distance between $\mathrm{X}$ and $\mathrm{H}$ are $\approx$ $9 \AA$ and $\approx 12 \AA$,respectively, and good agreement is obtained between the two methods. After introducing the solute atom(s), the ion positions are relaxed until the maximum force is less than $1 \mathrm{meV} / \AA$ while holding the dimensions of the supercell fixed at the pure Ni dimensions. To accurately obtain the misfit volume and misfit strain tensor, we performed calculations on various supercell sizes by duplicating the 4 -atoms unit cell to larger dimensions, $m_{1} \times m_{2} \times m_{3}$. After introducing the solute atom(s), all the ions are fully relaxed until the maximum force is less than $1 \mathrm{meV} / \AA$ while holding the lattices of the supercell fixed at the pure Ni values $\left(m_{i} a\right)$. For bulk Ni, although the supercells are constructed based on the stress-free unit cell, the stress tensors from the supercells of bulk $\mathrm{Ni}$ are quite small but not exactly zero. Therefore, in the following analysis, we eliminate effects of this stress by measuring only the stress tensor caused by the defect (i.e., $\boldsymbol{\sigma}_{X}-\boldsymbol{\sigma}_{N i}$ ). In Fig. 8, good linear relationships are observed between 
This is an Original Manuscript of an article published by Taylor \& Francis in Philosophical Magazine on 2017, available at http://www.tandfonline.com/doi/full/10.1080/14786435.2016.1263402.

the pressure (i.e. $-\frac{\sigma_{11}+\sigma_{22}+\sigma_{33}}{3}$ ) and the defect concentration. According to [23], the misfit volume of the defect can be obtained as

$$
\frac{\delta V}{V}=\frac{1}{B} \frac{\mathrm{d} p}{\mathrm{~d} c}
$$

where $B=196 \mathrm{GPa}$ and $\frac{\mathrm{d} p}{\mathrm{~d} c}$ are the bulk modulus of bulk Ni and the slope of the pressureconcentration curve, both obtained from our DFT calculations. For the misfit strain tensor, we choose one specific supercell, $3 \times 3 \times 3$, for all complexes and calculate the misfit strain associated with the measured stress state via Hooke's Law, i.e., $\overline{\boldsymbol{\varepsilon}}^{(\mathrm{m})}=\mathbf{C}^{-1} \boldsymbol{\sigma}$. The stresses are measured in a large cell whereas the misfit strain tensor associated with the primitive unit cell. Thus, the measured misfit strain is converted to the true misfit strain via

$$
\varepsilon^{(\mathrm{m})}=4 m_{1} m_{2} m_{3} \bar{\varepsilon}^{(\mathrm{m})} \text {. }
$$

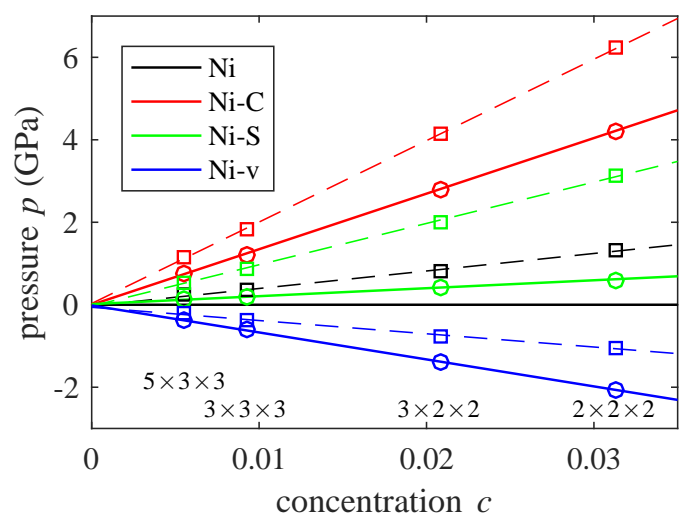

Figure 8.: Supercell pressure versus concentration for different solute complexes. Solid and dashed lines denote the cases with and without $\mathrm{H}$ atoms, respectively.

The computed misfit strain tensors and H-Solute binding energies are summarized in Table 2. For S substitutional solutes, the misfit strain tensor is volumetric and positive. The interaction energy of an $\mathrm{H}$ atom at octahedral site adjacent to this solute (see Fig. 9 ) is repulsive but relatively small $(0.022 \mathrm{eV})$. However, the misfit volume of the $\mathrm{S}-\mathrm{H}$ complex is $410 \%$ larger than that of a single $\mathrm{S}$. The $\mathrm{S}-\mathrm{H}$ misfit volume is also much larger $(62 \%)$ than the sum of the misfit volumes of isolated $\mathrm{S}$ and $\mathrm{H}$ interstitials by $62 \%$, indicating significant mechanical distortions. The larger misfit volume with low repulsion suggests that, in the presence of the stress field of the dislocation, $\mathrm{H}$ atoms could diffuse to the $\mathrm{S}$ atoms on the tension side of the dislocation core, where both $\mathrm{S}$ and $\mathrm{H}$ are already favorable, and overcome the repulsive energy to form $\mathrm{S}-\mathrm{H}$ complexes which have more misfit volume and thus are more favorable at the tensile side of the dislocation. The $\mathrm{S}-\mathrm{H}$ complex also has a tetragonal distortion, indicating that it can interact with a screw dislocation whereas the individual $\mathrm{H}$ and $\mathrm{S}$ atoms would not (away from the screw core). To quantify the tetragonal distortion versus the volume misfit, we define the distortion index $d$ as the ratio of the norm of the misfit distortion tensor to the volumetric strain tensor. Note that based on Eq. (8) the misfit strain of a tetragonal distorting solute can be written as

$$
\varepsilon^{\prime(m)}=\frac{\delta V^{(m)}}{3 V}\left(\frac{1-\xi}{1+2 \xi}\left(\begin{array}{ccc}
2 & 0 & 0 \\
0 & -1 & 0 \\
0 & 0 & -1
\end{array}\right)+\left(\begin{array}{ccc}
1 & 0 & 0 \\
0 & 1 & 0 \\
0 & 0 & 1
\end{array}\right)\right)
$$

Thus, $d=2\left(\frac{1-\xi}{1+2 \xi}\right)^{2}$ is the distortion index, with $d=0$ corresponding to volumetric only and $d \rightarrow \infty$ corresponding to isochoric distortion only. For the case of $\mathrm{S}-\mathrm{H}$ complex $d=0.0039$, 
This is an Original Manuscript of an article published by Taylor \& Francis in Philosophical Magazine on 2017, available at http://www.tandfonline.com/doi/full/10.1080/14786435.2016.1263402.

so the volumetric term dominates. For interstitial carbon, which occupies a octahedral site, the misfit volume is large. Adding a hydrogen interstitial on the tetrahedral site (see Fig. 9) increases the misfit volume $47 \%$, which is only slightly larger $(11.4 \%$ ) than the sum of the misfit volumes of a single $\mathrm{H}$ and $\mathrm{C}$ interstitials. However, the interaction energy of these two interstitials is repulsive and large $(\approx 0.73 \mathrm{eV})$, and so formation of this complex, even in the presence of the stress field of a dislocation, is not likely. The distortion index in this case is $d=0.012$. Furthermore the principal axes of the $\mathrm{C}-\mathrm{H}$ complex are not identical to the cubic direction.

Table 2.: Misfit strain tensor parameters for $\mathrm{S}$ and $\mathrm{C}$ atoms, and $\mathrm{SH}$ and $\mathrm{CH}$ complexes, in $\mathrm{Ni}$.

\begin{tabular}{lccccccc}
\hline solute & $\xi$ & $\eta$ & $\frac{\delta V}{V}$ & $\mathbf{e}_{1}^{\prime}$ & $\mathbf{e}_{2}^{\prime}$ & $\mathbf{e}_{3}^{\prime}$ & $E^{\text {int }}(\mathrm{eV})$ \\
\hline \hline $\mathrm{H}$ & 1.0 & 1.0 & 0.2168 & - & - & - & - \\
$\mathrm{S}$ & 1.0 & 1.0 & 0.0985 & - & - & - & - \\
$\mathrm{SH}$ & 0.877 & 0.877 & 0.5116 & {$[100]$} & {$[010]$} & {$[001]$} & 0.022 \\
$\mathrm{C}$ & 1.0 & 1.0 & 0.6882 & - & - & - & - \\
$\mathrm{CH}$ & 0.796 & 0.796 & 1.0082 & {$[\overline{1} \overline{1} 1]$} & {$[1 \overline{1} 0]$} & {$[112]$} & 0.734 \\
\hline
\end{tabular}

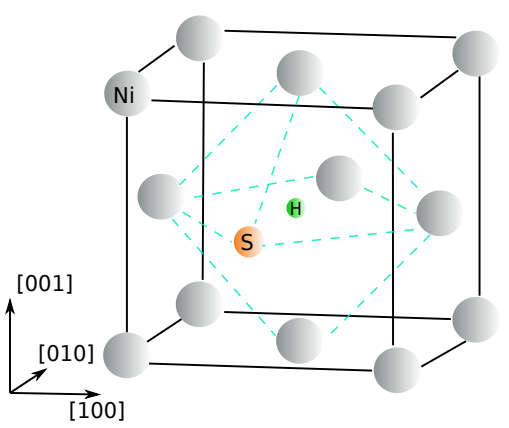

(a)

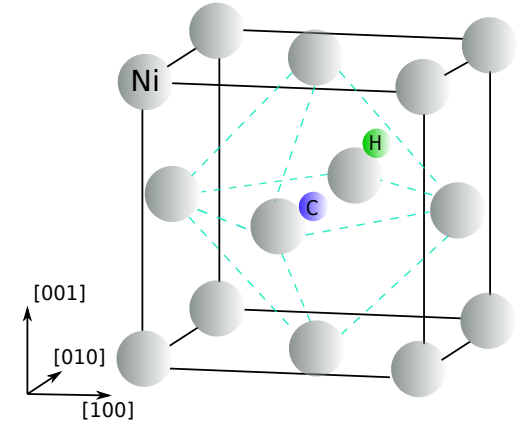

(b)

Figure 9.: Stable position of hydrogen interstitial in the vicinity of (a) a substitutional Sulfur atom and (b) an interstitial Carbon atom. Gray, dark blue, and orange spheres denote the $\mathrm{Ni}, \mathrm{C}$, and $\mathrm{S}$ atoms, respectively.

$\mathrm{S}$ atoms are a well-established embrittler in $\mathrm{Ni}$, being attracted to the grain boundaries. Cosegregation of $\mathrm{S}$ and $\mathrm{H}$ to the grain boundaries has been found to enhance embrittlement 41 . Thus examination of S-H complexes at grain boundaries is a natural direction for future work. Here we simply conclude that $\mathrm{S}$ and $\mathrm{H}$ repel in the bulk lattice, and that any formation of

300 an S-H complex would lead to an increased misfit strain and thus strengthening, rather than softening. For C, co-segregation of $\mathrm{H}$ and $\mathrm{C}$ is not expected. In both cases, the misfit distortion index is less than 0.04, whereas the $\mathrm{V}-\mathrm{H}$ complexes that show softening effects have $d=338$, so misfit distortions of SH and $\mathrm{CH}$ do not play a significant role in hardening or softening.

\section{Summary}

In summary, we have demonstrated that a softening effect (reduction in the stress required to move a dislocation) can be caused by the interaction of $\mathrm{H}$ atoms with solutes in metal alloys. The softening arises when the Solute- $\mathrm{H}$ complex leads to a reduction in the misfit strain tensor, and we have used vacancies as a surrogate solute in a Ni matrix to demonstrate the effect. The simulation results are consistent with the predictions of solute strengthening 
This is an Original Manuscript of an article published by Taylor \& Francis in Philosophical Magazine on 2017, available at http://www.tandfonline.com/doi/full/10.1080/14786435.2016.1263402.

theory, for both the scaling of the strength versus solute concentration and the magnitude of the softening. These results are perhaps the first atomistic simulations to support any mechanism for the proposed HELP theory, although connections to hydrogen embrittlement remain to be discovered. Solute-H strengthening is also possible, and is indicated here by detailed DFT computations for $\mathrm{S}-\mathrm{H}$ and $\mathrm{C}-\mathrm{H}$ complexes in Ni. Thus, this softening is not a

315 generic effect and will only arise in specific alloy systems. Our DFT analyses for S and C shows the operational path for assessing any solute of interest in any matrix materials. There are also other possible effects of $\mathrm{H}$ on plastic softening or strengthening that are not considered here. For instance, "solute drag" strengthening by relatively mobile H should serve as a generic strengthening mechanism. $\mathrm{H}$ interactions that modify solute interactions in the very core of

320 the dislocation may lead to either softening or strengthening; assessment of such possibilities would require extensive multiscale simulations 42 . H might influence dislocation/dislocation junction strengths, leading to strengthening or softening, similar to Dynamic Strain Aging in $\mathrm{Al}$ alloys 43 . In most of these situations, $\mathrm{H}$ transport appears necessary and so assessment of mechanisms must also address the kinetic aspects of $\mathrm{H}$ transport. The field thus remains rich

325 for exploration and with important implications for industrial applications of high strength metal alloys.

\section{Acknowledgments}

The authors gratefully acknowledge support of this work from the Swiss National Foundation through a grant for the project entitled "Predictive Mechanisms of Hydrogen Embrittlement" (project \# 200021-149207).

\section{References}

\section{References}

[1] W. H. Johnson, On some remarkable changes produced in iron and steels by the action of hydrogen acids, Proceedings of Royal Society of London 23 (1875) 168-175.

[2] R. L. S. Thomas, J. R. Scully, R. P. Gangloff, Internal hydrogen embrittlement of ultrahigh-strength aermet 100 steel, Metallurgical and Materials Transactions A: Physical Metallurgy and Materials Science 34 (2) (2003) 327-344.

URL www . scopus . com

[3] S. Bechtle, M. Kumar, B. P. Somerday, M. E. Launey, R. O. Ritchie, Grain-boundary engineering markedly reduces susceptibility to intergranular hydrogen embrittlement in metallic materials, Acta Materialia 57 (14) (2009) 4148-4157.

URL www . scopus . com

[4] D. H. Lassila, H. K. Birnbaum, JOM-J Miner Metal Mater Soc 36 (1984) 62. [link], URL www . scopus. com

[5] D. H. Lassila, H. K. Birnbaum, The effect of diffusive hydrogen segregation on fracture of polycrystalline nickel, Acta Metallurgica 34 (7) (1986) 1237-1243.

URL www . scopus. com

[6] Y. Lee, R. P. Gangloff, Measurement and modeling of hydrogen environment-assisted cracking of ultra-high-strength steel, Metallurgical and Materials Transactions A: Physical Metallurgy and Materials Science 38 A (13) (2007) 2174-2190.

URL www . scopus . com

[7] R. P. Gangloff, 6.02 - hydrogen-assisted cracking (2007).

URL www. scopus.com

[8] A. R. Troiano, The role of hydrogen and other interstitials in the mechanical behavior of metals, Trans.ASM 52 (1960) 54-80.

URL wWw . scopus. com

[9] W. W. Gerberich, P. Marsh, J. Hoehn, S. Venkateraman, H. Huang, T. Magnin, P. J. M. 
This is an Original Manuscript of an article published by Taylor \& Francis in Philosophical Magazine on 2017, available at http://www.tandfonline.com/doi/full/10.1080/14786435.2016.1263402.

Gras, Proc.Int.Conf.on Corrosion-Deformation Interactions (1993) 633[link],

URL www . scopus . com

[25] Y. N. Osetsky, D. J. Bacon, An atomic-level model for studying the dynamics of edge dislocations in metals, Modelling and Simulation in Materials Science and Engineering 11 (4) (2003) 427-446. doi:10.1088/0965-0393/11/4/302.

410

[10] R. A. Oriani, P. H. Josephic, Equilibrium aspects of hydrogen-induced cracking of steels Acta Metallurgica 22 (9) (1974) 1065-1074. URL www . scopus . com

[11] W. W. Gerberich, T. J. Foeche, Hydrogen Enhanced Decohesion in Fe-Si Single Crystals: Implications to Modelling of Thresholds (1990) 687[link] URL www . scopus . com

[12] R. A. Oriani, A mechanistic theory of hydrogen embrittlement of steels Ber.Bunsenges.Phys.Chem. 76 (8) (1972) 848-857. URL www . scopus . com

[13] W. W. Gerberich, P. G. Marsh, J. W. Hoehn, Hydrogen induced cracking mechanisms are there critical experiments?, in: Hydrogen Effects in Materials, 1996, pp. 539-551. URL www. scopus.com

[14] J. Song, W. A. Curtin, A nanoscale mechanism of hydrogen embrittlement in metals Acta Materialia 59 (4) (2011) 1557-1569, cited By :36. URL www. scopus . com

[15] J. Song, W. A. Curtin, Atomic mechanism and prediction of hydrogen embrittlement in iron, Nature Materials 12 (2) (2013) 145-151, cited By :21.

URL www . scopus . com

[16] B. CD, New model for hydrogen- assisted cracking (hydrogen embrittlement), Met Trans 3 (2) (1972) 437-451.

URL www . scopus . com

[17] H. K. Birnbaum, P. Sofronis, Hydrogen-enhanced localized plasticity-a mechanism for hydrogen-related fracture, Materials Science and Engineering A 176 (1-2) (1994) 191202.

URL www . scopus . com

[18] I. M. Robertson, The effect of hydrogen on dislocation dynamics, Engineering Fracture Mechanics 68 (6) (2001) 671-692.

URL www . scopus .com

[19] J. Song, W. Curtin, Mechanisms of hydrogen-enhanced localized plasticity: an atomistic study using $\alpha$-fe as a model system, Acta Materialia 68 (2014) 61-69.

[20] J. von Pezold, L. Lymperakis, J. Neugebeauer, Hydrogen-enhanced local plasticity at dilute bulk $\mathrm{h}$ concentrations: The role of $\mathrm{h}-\mathrm{h}$ interactions and the formation of local hydrides, Acta Materialia 59 (8) (2011) 2969-2980.

[21] G. P. M. Leyson, W. A. Curtin, L. G. Hector, C. F. Woodward, Quantitative prediction of solute strengthening in aluminium alloys, Nature Materials 9 (9) (2010) 750-755, cited By :43.

URL www . scopus . com

[22] G. P. M. Leyson, L. G. Hector Jr., W. A. Curtin, First-principles prediction of yield stress for basal slip in mg-al alloys, Acta Materialia 60 (13-14) (2012) 5197-5203, cited By :11. URL www . scopus .com

[23] G. P. M. Leyson, L. G. Hector Jr., W. A. Curtin, Solute strengthening from first principles and application to aluminum alloys, Acta Materialia 60 (9) (2012) 3873-3884, cited By $: 21$.

URL www . scopus .com

[24] M. Hestenes, E. Stiefel, Methods of conjugate gradients for solving linear systems, Journal of Research of the National Bureau of Standards 49 (6) (1952) 409. doi:10.6028/jres. 049.044

[26] T. Schneider, E. Stoll, Molecular-dynamics study of a three-dimensional one-component model for distortive phase transitions, Physical Review B 17 (3) (1978) 1302-1322. 
This is an Original Manuscript of an article published by Taylor \& Francis in Philosophical Magazine on 2017, available at http://www.tandfonline.com/doi/full/10.1080/14786435.2016.1263402.

[27] P. H. B. William C. Swope Hans C. Andersen, K. R. Wilson, A computer simulation method for the calculation of equilibrium constants for the formation of physical clusters of molecules: Application to small water clusters, J. Chem. Phys. 76 (1) (1982) 637-649. doi:10.1063/1.442716 URL http://jcp.aip.org/resource/1/jcpsa6/v76/i1/p637_s1

[28] J. E. Angelo, N. R. Moody, M. I. Baskes, Trapping of hydrogen to lattice defects in nickel, Modelling and Simulation in Materials Science and Engineering 3 (3) (1995) 289307, cited By :150. URL www . scopus. com

[29] S. Plimpton, Fast parallel algorithms for short-range molecular dynamics, Journal of computational physics 117 (1) (1995) 1-19.

[30] A. Stukowski, Visualization and analysis of atomistic simulation data with ovito-the open visualization tool, Modelling and Simulation in Materials Science and Engineering 18 (1) (2009) 015012.

[31] G. Leyson, L. Hector, W. Curtin, Solute strengthening from first principles and application to aluminum alloys, Acta Materialia 60 (9) (2012) 3873-3884.

[32] G. Kresse, J. Furthmüller, Efficient iterative schemes for ab initio total-energy calculations using a plane-wave basis set, Physical Review B 54 (16) (1996) 11169-11186. doi:10. 1103/PhysRevB.54.11169.

URL http://link.aps .org/doi/10.1103/PhysRevB.54.11169

[33] G. Kresse, From ultrasoft pseudopotentials to the projector augmented-wave method, Physical Review B 59 (3) (1999) 1758-1775. doi:10.1103/PhysRevB.59.1758.

[34] J. P. Perdew, K. Burke, M. Ernzerhof, Generalized Gradient Approximation Made Simple, Physical Review Letters 77 (18) (1996) 3865-3868. arXiv:0927-0256 (96) 00008 , doi:10.1103/PhysRevLett.77.3865.

URL http://www.ncbi.nlm.nih.gov/pubmed/10062328\$/delimiter"026E30F\$nhttp: //link.aps.org/doi/10.1103/PhysRevLett.77.3865\$delimiter"026E30F\$nhttp: //link.aps.org/abstract/PRL/v77/p3865\$delimiter"026E30F\$nhttp://link. aps.org/doi/10.1103/PhysRevLett.77.3865

[35] P. E. Blöchl, Projector augmented-wave method, Physical Review B 50 (24) (1994) 1795317979. arXiv:arXiv:1408.4701v2, doi:10.1103/PhysRevB.50.17953

[36] M. Methfessel, A. T. Paxton, High-precision sampling for Brillouin-zone integration in metals, Physical Review B 40 (6) (1989) 3616-3621. doi:10.1103/PhysRevB.40.3616.

[37] H. J. Monkhorst, J. D. Pack, Special points for Brillouin-zone integrations, Physical Review B 13 (12) (1976) 5188-5192. doi:10.1103/PhysRevB.13.5188.

[38] G. Simmons, H. Wang, et al., Single crystal elastic constants and calculated aggregate properties, Mass., MIt Press, 1971.

[39] G. Alers, J. Neighbours, H. Sato, Temperature dependent magnetic contributions to the high field elastic constants of nickel and an fe-ni alloy, Journal of Physics and Chemistry of Solids 13 (1) (1960) 40-55.

[40] M. B. Stearns 19 (1986) 24.

[41] D. H. Lassila, H. K. Birnbaum, Intergranular Fracture of Hydrogen-Sulfur of Nickel : the Effect of hydrogen-sulfur co-segregeation, Acta Metallurgica 35 (7). doi:10.1016/ 0001-6160 (87) 90127-1.

[42] L. Ventelon, B. Lüthi, E. Clouet, L. Proville, B. Legrand, D. Rodney, F. Willaime, Dislocation core reconstruction induced by carbon segregation in bcc iron, Physical Review B 91 (22) (2015) 220102.

[43] M. Soare, W. Curtin, Solute strengthening of both mobile and forest dislocations: The origin of dynamic strain aging in fcc metals, Acta Materialia 56 (15) (2008) 4046-4061. 